\title{
Do the Medial Temporal Lobes Resolve Perceptual Interference?
}

\author{
Bronson Harry, ${ }^{1}$ Mark Postans, ${ }^{2}$ and Katja Umla-Runge ${ }^{2}$ \\ ${ }^{1}$ School of Psychology, Bangor University, LL57 2AS Bangor, United Kingdom, and ${ }^{2}$ School of Psychology, Cardiff University, CF10 3AT Cardiff, United \\ Kingdom \\ Review of Watson and Lee.
}

The role of the medial temporal lobes (MTLs) in memory was first clearly established with the case of patient HM, who suffered from an inability to form new memories following surgical removal of bilateral MTLs (Scoville and Milner, 1957). Subsequent work in humans and animals revealed that memory impairment associated with MTL damage was related to a set of subcortical and cortical structures including the hippocampus, entorhinal, perirhinal, and parahippocampal cortex. Recent studies have shown that some of these subregions are domain sensitive with evidence indicating that the parahippocampal gyrus and hippocampus are involved in scene recognition, whereas the perirhinal cortex is involved in recognition of individual objects (Taylor et al., 2007; Staresina et al., 2011). Despite these considerable advances in our understanding of this brain region over the last 60 years, there remain unresolved questions regarding the fundamental role the MTL plays in perception and memory.

One current controversy rests on evidence that MTL damage impairs perceptual discrimination in tasks that place no

Received April 26, 2013; revised May 25, 2013; accepted May 30, 2013.

This work was supported by the Biotechnology and Biological Sciences Research Council Grant BB/1007091/1. We thank Nils Muhlert and an anonymous reviewer for providing comments on an earlier version of the manuscript.

Correspondence should be addressed to Dr. Bronson Harry, School of Psychology, Brigantia Building, Bangor University, LL57 2AS Bangor, United Kingdom. E-mail: b.harry@bangor.ac.uk.

DOI:10.1523/JNEUROSCI.1767-13.2013

Copyright $\odot 2013$ the authors $\quad 0270-6474 / 13 / 3310935-03 \$ 15.00 / 0$ or minimal demands on memory. Many studies have reported domain-specific patterns of perceptual impairment following damage to different MTL subregions with object and scene discrimination impairments associated with perirhinal and hippocampal damage respectively (Lee et al., 2005; Saksida and Bussey, 2010). Importantly, the perceptual deficits reported in these studies were only apparent under conditions where stimuli shared many features, suggesting that MTL subregions differentially support complex representations that are recruited to distinguish highly similar objects and scenes. Evidence that MTL damage impairs high-level vision seemingly conflicts with the notion that the MTL is a "memory system" that has no role in cognition outside the mnemonic domain.

Under representational accounts of MTL function, amnesia associated with MTL damage is reframed as a representational deficit where forgetting occurs due to a failure in resolving perceptual interference (Bartko et al., 2010; Graham et al., 2010; Barense et al., 2012). These models borrow ideas from interference theories of amnesia (Warrington and Weiskrantz, 1974) which propose that successful recognition judgments occur when there is sufficient overlap in the features perceived during initial and subsequent encounters with a stimulus. According to this view, nonamnesic forgetting occurs due to overlapping features between a target stimulus and nontarget (foil) stimuli seen during the retention interval. This inter- ference weakens memory for the original target stimulus. Representation-based accounts of amnesia argue that the MTL buffers recognition memory against interference effects, by forming and maintaining the complex perceptual representations, which distinguish stimuli that share features. Damage to the MTL affects the complex representations that distinguish similar stimuli so that recognition judgments become reliant on simple features, such as lines, curves and edges, which are associated with less complex representations in occipito-temporal cortex. Because such features are not uniquely associated with one particular stimulus, perceptual interference will rapidly accumulate leading to chronic amnesia.

A recent report by Watson and Lee (2013) provides an important test of the idea that MTL regions are involved in resolving perceptual interference. The authors devised a novel interference matchto-sample task consisting of compound scene and object images, which they used to assess whether different MTL regions were involved in resolving scene and object interference effects in healthy participants. Each trial of the task involved three phases: exposure, interference, and test.

In the exposure phase, participants were presented with a single compound image consisting of a common object (e.g., camera) superimposed on a computer generated indoor scene (Watson and Lee, 2013; their Fig. 1). 
In the interference phase in between encoding and test, seven object-compound images were presented. In the objectinterference condition, seven different object exemplars (e.g., seven different camera models), which were perceptually similar to the encoding stimulus, were presented in front of the unchanged scene. In the scene interference condition, seven scenes that were perceptually similar in texture and surface to the encoding stimulus were presented together with the unchanged object. With this method, interference was induced for only one of the two components of the compound images.

In the test phase, participants determined which of two simultaneously presented compound images (target and foil) were identical to the exposure image. Importantly, the target and foil image were perceptually similar, ensuring that successful recognition would (by hypothesis) require the complex representations stored in the MTL. Each trial involved either object or scene recognition, which was determined by varying only object or only scene information in the foil image. For example, on object recognition trials, the scene component in both the target and the foil matched that of the exposure image, whereas the object component matched that in the exposure image only in the target, not in the foil. Participants were required to retain both scene and object information in memory during the interference phase because the type of recognition judgment required on each trial was not apparent until the test phase, and each type of recognition judgment could follow each type of interference (e.g., object recognition following scene interference).

The rationale behind the task was that the perceptual interference generated in the retention interval would increase demand on MTL regions when participants attempted to match the target and exposure image (i.e., at test). The authors hypothesized that target recognition (in the presence of perceptual interference) would involve accessing the complex MTL representations to distinguish the memory of the exposure image from that of the perceptually similar interference stimuli. Moreover, the authors predicted that object-sensitive MTL regions (e.g., perirhinal cortex) would show increased activity only for object recognition/object interference trials whereas scene-sensitive MTL regions (e.g., parahippocampal gyrus, hippocampus) would show increased activity only for scene recognition/scene interference trials. Indeed, the behavioral data showed a domainspecific pattern of interference with impaired object recognition judgments that were preceded by object interference, and impaired scene recognition judgments that were preceded by scene interference (Watson and Lee, 2013, their Fig. 2).

Importantly, fMRI results revealed that the left perirhinal cortex was more active during object recognition judgements that were preceded by object interference compared with object recognition judgements that were preceded by scene interference, and to scene recognition judgments that were preceded by either object or scene interference (Watson and Lee, 2013, their Fig. 3b). This pattern of results fits with the hypothesis that this region supports high-level representations that are involved in distinguishing the memory of the exposure image from the perceptually similar interference objects.

Although the results showed that the perirhinal cortex was involved in resolving object interference, neither of the scenesensitive MTL brain regions (parahippocampal gyrus, hippocampus) showed the corresponding pattern of results for scene recognition trials. Instead of showing a selective increase in activity for scene recognition/scene interference trials, the parahippocampal gyrus showed a general increase in activity for scene recognition trials (compared with object recognition trials) regardless of whether object or scene interference appeared in the retention interval (Watson and Lee, 2013, their Fig. 5b). A general increase in activity for all scene recognition judgments indicates that the parahippocampal gyrus does not contain the complex scene representations that are selectively involved in distinguishing the memory of the exposure image from the perceptually similar interference scenes. Instead, these results suggest that the parahippocampal gyrus may contain general representations that are involved in all scene recognition judgments. The parahippocampal gyrus also showed increased activity for scene interference trials (compared with object interference trials) regardless of whether participants performed a scene or object recognition judgment. The authors speculated that reduced activity on object interference trials might reflect scene-related repetition suppression, since the object interference condition involved repeatedly presenting the same scene image, whereas the scene interference condition involved presenting different scene images. Moreover, the hippocampus showed inhibition only for scene recognition trials that were preceded by object interference (Watson and Lee, 2013; their Fig. $4 b)$. This pattern of results is difficult to ex- plain with a hypothesis based on either object or scene-based interference resolution. The authors point out that the hippocampus is involved in a range of processes that are not specific to either objects or scenes (e.g., Kumaran and Maguire, 2007) and so it is possible that an interaction with these could mask scene selective interference effects during scene recognition. However, this suggestion cannot be answered based on the existing dataset and would need further empirical testing.

A potential limitation of this study concerns whether the changes in perirhinal cortex activity induced by the interference phase on object recognition trials resulted from changes in decay of object information across interference conditions. On scene interference trials, the target object appeared in the exposure, interference and test phases whereas the target object only appeared at exposure and test on object interference trials. So it might be the case the object interference trials were associated with increased decay of target object information. If this were the case, then the observed increase in perirhinal cortex activity for object recognition/object interference trials might have been related to a larger decay of the target on these trials. One possible solution to this limitation would be to present images of objects from a different category to the target object in the interference phase. Objects from a different category are less likely to share features with the target image, and so should induce less perceptual interference. In this way, the target image would only appear in the exposure and target phase for both high and low interference conditions.

However, we must point out that repeatedly presenting the same object is associated with one advantage in that absolutely no perceptual interference could accumulate during the interference phase. Some perceptual interference may accumulate even when objects from a different category are used in the interference phase if there is some level of feature overlap between the target and distracter category. We suggest that future studies should include repeatedimage and different-category interference trials to distinguish between decay and interference effects.

Notwithstanding considerations regarding how perceptual interference was implemented, the interference match to sample task provides a valuable tool to help explore representational accounts of the MTL and may uncover how different regions contribute to perception and memory. Importantly, this technique 
promises to offer new insights into how forgetting occurs in the healthy brain and may open the way to a better understanding of memory disorders.

\section{References}

Barense MD, Groen II, Lee AC, Yeung LK, Brady SM, Gregori M, Kapur N, Bussey TJ, Saksida LM, Henson RN (2012) Intact memory for irrelevant information impairs perception in amnesia. Neuron 75:157-167. CrossRef Medline

Bartko SJ, Cowell RA, Winters BD, Bussey TJ, Saksida LM (2010) Heightened susceptibility to interference in an animal model of amnesia: Impairment in encoding, storage, retrieval—or all three? Neuropsychologia 48:2987-2997. CrossRef Medline

Graham KS, Barense MD, Lee AC (2010) Going beyond LTM in the MTL: a synthesis of neuropsychological and neuroimaging findings on the role of the medial temporal lobe in memory and perception. Neuropsychologia 48:831-853. CrossRef Medline

Kumaran D, Maguire EA (2007) Match-mismatch processes underlie human hippocampal responses to associative novelty. J Neurosci 27 : 8517-8524. CrossRef Medline

Lee AC, Bussey TJ, Murray EA, Saksida LM, Epstein RA, Kapur N, Hodges JR, Graham KS (2005) Perceptual deficits in amnesia: challenging the medial temporal lobe "mnemonic" view. Neuropsychologia 43:1-11. CrossRef Medline

Saksida LM, Bussey TJ (2010) The representational-hierarchical view of amnesia: translation from animal to human. Neuropsychologia 48: 2370-2384. CrossRef Medline

Scoville WB, Milner B (1957) Loss of recent memory after bilateral hippocampal lesions. J Neurol Neurosurg Psychiatry 20:11-21. CrossRef Medline

Staresina BP, Duncan KD, Davachi L (2011) Perirhinal and parahippocampal cortices differentially contribute to later recollection of object- and scene-related event details. J Neurosci 31:8739-8747. CrossRef Medline

Taylor KJ, Henson RN, Graham KS (2007) Recognition memory for faces and scenes in amnesia: dissociable roles of medial temporal lobe structures. Neuropsychologia 45:2428-2438. CrossRef Medline

Warrington EK, Weiskrantz L (1974) The effect of prior learning on subsequent retention in amnesic patients. Neuropsychologia 12:419428. CrossRef Medline

Watson HC, Lee AC (2013) The perirhinal cortex and recognition memory interference. J Neurosci 33:4192-4200. CrossRef Medline 